Check for updates

Cite this: J. Mater. Chem. A, 2020, 8 , 21872

Received 1st July 2020

Accepted 10th October 2020

DOI: $10.1039 / d 0 t a 06428 a$

rsc.li/materials-a

\section{Mechanisms of the cyclic (de)lithiation of $\mathrm{RuO}_{2} \dagger$}

\author{
Lin Xu (D) and Carl V. Thompson (D)*
}

$\mathrm{RuO}_{2}$ films can serve as high-performance electrodes for thin film lithium-ion batteries due to their large volumetric charge capacity, low rate sensitivity and excellent cyclability. Unlike other electrode materials, $\mathrm{RuO}_{2}$ films also do not require high temperature processing, making them suitable for integration with low-power CMOS circuits and for fabrication on flexible membranes. To determine the mechanisms through which $\mathrm{Li}$ is reversibly stored in $\mathrm{RuO}_{2}$ films, detailed characterization studies of sputtered thin films were carried out; galvanostatic and potentiostatic intermittent titration and cyclic voltammetry studies were coupled with ex situ selected area electron diffraction, X-ray photoelectron and Raman spectroscopy, optical and scanning electron microscopy, energy-dispersive $\mathrm{X}$-ray spectroscopy and in situ electrochemical impedance spectroscopy. During lithiation, amorphous $\mathrm{RuO}_{2}$ is transformed to amorphous $\mathrm{Li}_{x} \mathrm{RuO}_{2}$ through an alloying reaction and this is followed by a reversible side reaction to form an SEl layer. $\mathrm{Li}_{x} \mathrm{RuO}_{2}$ then undergoes a conversion reaction to form a mixture of nanosized $\mathrm{Ru}$ and $\mathrm{Li}_{2} \mathrm{O}$ crystals, and finally at low voltages $\mathrm{Li}$ is inserted into the $\mathrm{Ru} / \mathrm{Li}_{2} \mathrm{O}$ mixture. These reactions occur in a different sequence during delithiation and a large overpotential is required to reverse the conversion reaction, leading to a large energy loss during cycling. It is argued that this hysteretic behavior is associated with slow diffusive processes required for the conversion reactions. The methodology developed in this study can also be applied to other candidate thin film electrode materials and learnings from studies of thin films can be applied to more complex powder-based electrodes used in bulk batteries.

\section{Introduction}

While the use of lithium ion batteries (LIBs) is already pervasive, the range of applications for LIBs continues to rapidly grow, especially in association with the development of new Internet of Things (IoT) technologies. ${ }^{\mathbf{1}}$ The broad extent and diversity of applications of LIBs drive studies for continuous improvements in the performance of known electrode materials and development of new materials. Electrode materials that store lithium through phase transitions and conversion reactions are attractive candidates for LIB electrodes due to their large capacities compared to intercalation materials. ${ }^{2-11}$ For metal oxides, a conversion reaction plays a particularly important role in providing high storage capacity. This reaction has the form:

$$
\mathrm{M}_{a} \mathrm{X}_{b}+(b n) \mathrm{Li} \leftrightarrow a \mathrm{M}+b \mathrm{Li}_{n} \mathrm{X}
$$

Recently, Perego et al. ${ }^{\mathbf{1 2}}$ demonstrated that one conversionreaction-based material, $\mathrm{RuO}_{2}$, had excellent performance as a potential cathode material for thin film LIBs for microsystems, for which full-cell voltages as low as $1 \mathrm{~V}$ are usable. RF-

Department of Materials Science and Engineering, Massachusetts Institute of Technology, Cambridge, Massachusetts 02139, USA. E-mail: cthomp@mit.edu

$\uparrow$ Electronic supplementary information (ESI) available. See DOI: 10.1039/d0ta06428a sputtered $\mathrm{RuO}_{2}$ thin films showed five times greater volumetric energy than RF-sputtered $\mathrm{LiCoO}_{2}$ thin films. Moreover, unlike other electrode materials, $\mathrm{RuO}_{2}$ films do not require high temperature processing, making them suitable for integration with low-power CMOS circuits and for fabrication on flexible membranes. The mechanical behavior during the lithiation/ delithiation cycling of $\mathrm{RuO}_{2}$ thin films has been studied by Zhu et al. ${ }^{13,14}$ However, lithiation and delithiation mechanisms for multiply-cycled $\mathrm{RuO}_{2}$ are poorly understood due to the poor cyclability of powder-based electrodes, ${ }^{15}$ and an improved understanding is required for optimization of battery performance and yield of thin film electrodes.

The lithiation of $\mathrm{RuO}_{2}$ was first studied by Ohzuku et al., ${ }^{16}$ and the mechanisms for Li storage have subsequently been studied in various ways, especially during the first discharge of single crystal particles. David et al. studied the shallow lithiation of $\mathrm{RuO}_{2}$, before the occurrence of conversion reaction. ${ }^{\mathbf{1 7}}$ Balaya et al. ${ }^{15,18}$ used X-ray diffraction (XRD), Raman spectroscopy, high resolution transmission electron microscopy (HRTEM) and selected area electron diffraction (SAED) to show that crystalline $\mathrm{RuO}_{2}$ could form crystalline $\mathrm{Ru}$ and $\mathrm{Li}_{2} \mathrm{O}$ during the first lithiation and returned to nanocrystalline or amorphous $\mathrm{RuO}_{2}$ after the first delithiation. Gregorczyk et al. ${ }^{19}$ used in situ TEM and found that an intermediate phase $\mathrm{Li}_{x} \mathrm{RuO}_{2}$ formed before the formation of Ru during the first lithiation. They also reported that during further cycles of a nanowire, the reaction between amorphous $\mathrm{RuO}_{2}$ and $\mathrm{Ru} / \mathrm{Li}_{2} \mathrm{O}$ was only 
partially reversible. The existence of the intermediate phase $\mathrm{Li}_{x} \mathrm{RuO}_{2}$ was also confirmed by Mao et al. ${ }^{20}$ using in situ TEM. Hassan et al. ${ }^{21}$ used first-principles calculations to investigate the first lithiation of $\mathrm{RuO}_{2}$ and argued that additional $\mathrm{Li}$ could reside at interfaces between $\mathrm{Ru}$ and $\mathrm{Li}_{2} \mathrm{O}$. Hu et al. ${ }^{22}$ used XRD, XAS (X-ray absorption spectroscopy) and NMR (nuclear magnetic resonance) to demonstrate that the formation of $\mathrm{Li}_{2} \mathrm{O}$ and $\mathrm{LiH}$ from $\mathrm{LiOH}$ on the surface was responsible for the additional capacity of $\mathrm{RuO}_{2}$ during the late stage of the first lithiation, while based on TEM, XPS and GITT (Galvanostatic Intermittent Titration Techniques) experiments, Kim et al. ${ }^{23}$ argued that $\mathrm{Li}$ storage at interfaces between newly formed nanosized $\mathrm{Ru}$ metal and $\mathrm{Li}_{2} \mathrm{O}$ phases was responsible for the additional capacity. Emilie et al. reached the same conclusion using ${ }^{6} \mathrm{Li}$ magic-angle spinning nuclear magnetic resonance. ${ }^{24}$

So far, most studies of the mechanisms of lithiation and delithiation of $\mathrm{RuO}_{2}$ have focused only on the first lithiation process starting from crystalline $\mathrm{RuO}_{2}$ powders in composite electrodes with binders. This might be partially due to the poor cyclability of these electrodes. ${ }^{15}$ However, an in situ TEM study ${ }^{19}$ using $\mathrm{RuO}_{2}$ nanowires has shown that after the first delithiation cycle, the $\mathrm{RuO}_{2}$ was in an amorphous form rather than a crystalline form. This indicates that the previous conclusions reached from studies of the first cycle do not necessarily apply during further cycles. Electrochemical data such as cyclic voltammetry (CV) curves were not provided for cycles after the first cycle. In addition, conversion-type LIB electrodes are often found to have a large voltage hysteresis, meaning that the voltage of charging (delithiation) is significantly higher than the voltage of discharging (lithiation). ${ }^{\mathbf{9} 10,25-29}$ The voltage hysteresis for conversion-type LIB electrodes is generally larger than for intercalation $^{30}$ and alloying ${ }^{31}$ electrodes. We found that $\mathrm{RuO}_{2}$ also has a large voltage hysteresis (Fig. S1†).

As a good candidate for thin-film electrode materials as well as an example of a conversion-reaction-based electrode material with complex reaction mechanisms, it is important that a more complete understanding of the mechanisms and kinetics of reversible storage of $\mathrm{Li}$ in $\mathrm{RuO}_{2}$ be developed, not only in the first cycles, but also during subsequent cycles. In many ways, thin films also provide an ideal form for mechanistic studies. Therefore, while studies of thin film electrodes serve to guide optimization for use in thin film batteries, they also provide a guide for development of conversion-reaction powder electrodes with improved cyclability and reduced hysteretic effects.

In this work, we performed detailed electrochemical and physical characterization of multiply-cycled sputtered thin film $\mathrm{RuO}_{2}$ samples. Individual reactions occurring in different voltage regimes were identified through a set of GITT and CV measurements designed for this purpose. Samples were then characterized in different states of charge using ex situ Selected Area Electron Diffraction (SAED), X-ray Photoelectron Spectroscopy (XPS), Raman spectroscopy, Optical Microscopy (OM), Scanning Electron Microscopy (SEM), Energy-dispersive X-ray Spectroscopy (EDS) and in situ Electrochemical Impedance Spectroscopy (EIS). The Potentiostatic Intermittent Titration Technique (PITT) and CV measurements on $\mathrm{RuO}_{2}$ thin films with different thicknesses were also performed to determine the rate limiting kinetic process that leads to the voltage hysteresis.

\section{Experimental}

Double-side polished aluminum oxide (99.6\%, $\mathrm{Al}_{2} \mathrm{O}_{3}, 250 \mu \mathrm{m}$ thick, Stellar Ceramics) was used as the substrate for $\mathrm{RuO}_{2}$ thin film deposition. The aluminum oxide was cleaved into $5 \mathrm{~mm}$ by $8 \mathrm{~mm}$ pieces and was subsequently ultrasonicated for $5 \mathrm{~min}$ in acetone, isopropyl alcohol and deionized water to remove surface contaminants. After this step, the substrates were rinsed again in deionized water and dried using nitrogen. A $10 \mathrm{~nm}$ thick titanium film was deposited on the alumina to serve as an adhesion layer and a $100 \mathrm{~nm}$-thick palladium film was then deposited to serve as a current collector. $\mathrm{RuO}_{2}$ thin films were sputtered (CMS-18 Kurt J. Lesker) onto the Pd layer under 3 mTorr of pure $\mathrm{O}_{2}(99.994 \%$, Airgas) and at a $60 \mathrm{~W}$ RF power at room temperature.

To prepare half cells, samples were assembled into customized cells (Tomcell, Japan). Metallic Li was used as the counter electrode. The liquid electrolyte was standard LP30 electrolyte (1 $\mathrm{M} \mathrm{LiPF}_{6}$ in $1: 1$ ethylene carbonate and dimethyl carbonate mixture by volume, Gotion). Electrodes were separated using a porous polymer separator (Celgard). Electrochemical experiments (GITT/CV/EIS/PITT) were performed using a Solartron $1470 \mathrm{E}$ potentiostat. After samples were cycled 10 times and the CV curves became stable, in situ EIS was performed before disassembly of the cells.

The cells were disassembled and the cycled thin film samples were rinsed in dehydrated dimethyl carbonate (DMC) to remove residual electrolyte on the surface, and then dried using flowing ultrapure Ar, while still in a glovebox. The clean samples were placed in an air-tight container that was evacuated in the glove box and transferred to a dual-beam SEM/FIB system (Helios Nanolab 600, FEI), with approximately a oneminute exposure to ambient during loading. SEM/EDS results were then collected, and cross sectional TEM samples were prepared. The TEM samples were then transferred to a $200 \mathrm{kV}$ TEM (JEOL 2010), during which they were again exposed to ambient for approximately one minute. SAED patterns were collected in the TEM.

The remaining portions of the thin film samples from which TEM samples were prepared were transferred back to the glovebox (approximately a three-minute ambient exposure) and subsequently to a chamber for characterization using XPS (Versaprobe II, PHI) under vacuum, with another five-minute exposure to ambient during sample loading. After XPS measurements, Raman spectroscopy (InVia Reflex Micro Raman, Renishaw) was performed in ambient.

\section{Results and discussion}

\subsection{GITT and CV scans}

As discussed above, in previous studies CV scans have only been reported for very early cycles of initially crystalline $\mathrm{RuO}_{2}$ electrodes. Here we first report the stable $\mathrm{CV}$ curves of thin film $\mathrm{RuO}_{2} / \mathrm{LiPF}_{6} / \mathrm{Li}$ half-cells after over 10 cycles. These $\mathrm{RuO}_{2}$ films 
were deposited using RF magnetron sputtering at room temperature and experiments were carried out on films in the as-deposited state, which was found to initially be nanocrystalline. The CV curves display different behaviors in the $1^{\text {st }}$ cycle compared to later cycles, but after 10 cycles the shapes and magnitudes of peaks become highly repeatable (Fig. S2 $\dagger$ ). A typical stable $\mathrm{CV}$ curve for $\mathrm{RuO}_{2}$ is shown in Fig. 1. As can be seen in this figure, during discharge (lithiation), two clear reaction peaks and a shoulder are present, which indicates the existence of at least three different reactions. On the other hand, four peaks can be seen during charge (delithiation), indicating at least four different reactions. This implies that overlapping peaks appear as one peak during lithiation. For convenience in later discussions, the $\mathrm{CV}$ peaks are labeled as shown in Fig. 1 so that the mechanisms identified with individual peaks can be described more compactly.

In Fig. 1, the shoulder on the main lithiation peak was tentatively identified as corresponding to an independent reaction $\beta$. To confirm this, GITT tests were carried out. During GITT tests, the equilibrium voltages of materials at specific states of charge can be obtained after long relaxation steps, minimizing the influence of kinetic effects. When the differences in the state of charge $\Delta x$ between two consecutive steps are kept the same, a small change in the corresponding equilibrium voltage $\Delta V$ indicates that a reaction has occurred. Peaks in plots of $1 / \Delta V$ vs. $V$ are therefore related to peaks seen in $\mathrm{CV}$ curves. However, because relaxation is allowed in GITT tests, kinetic effects that can cause overlap of peaks in CV curves are reduced.

The $1 / \Delta V v s$. voltage curves extracted from GITT tests in a Li/ $\mathrm{RuO}_{2}$ cell are shown in Fig. 2a, and a direct comparison with a CV curve is shown in Fig. 2b. Here it can be clearly seen that the same four delithiation peaks appear in both the GITT and

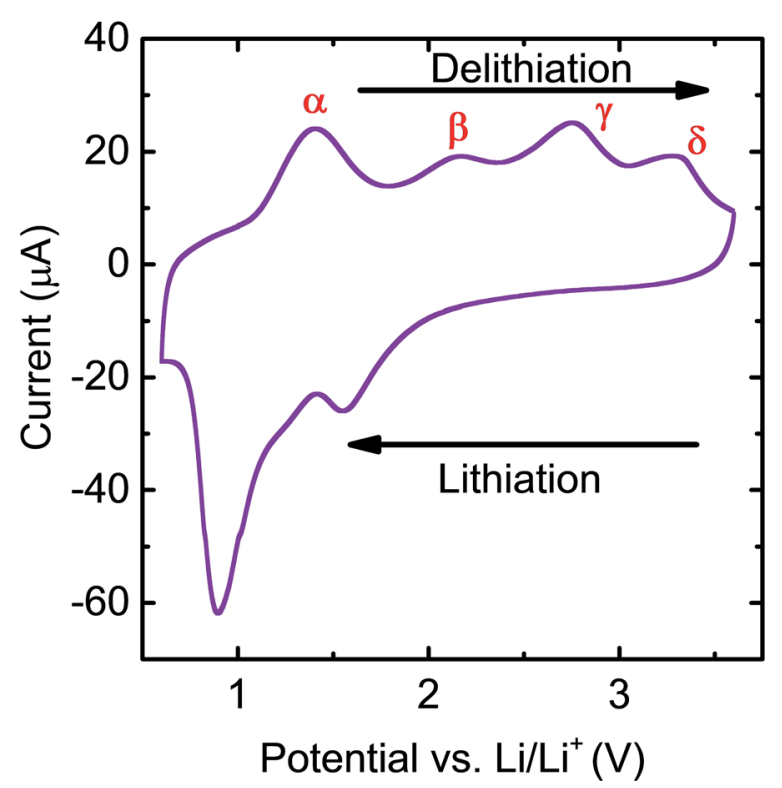

Fig. 1 Cyclic voltammogram for an $\mathrm{RuO}_{2} / \mathrm{LiPF}_{6} / \mathrm{Li}$ cell obtained at a scan rate of $0.5 \mathrm{mV} \mathrm{s}^{-1}$ between $0.6 \mathrm{~V}$ and $3.6 \mathrm{~V}$. The rationale for the peak labels is explained in the main text.
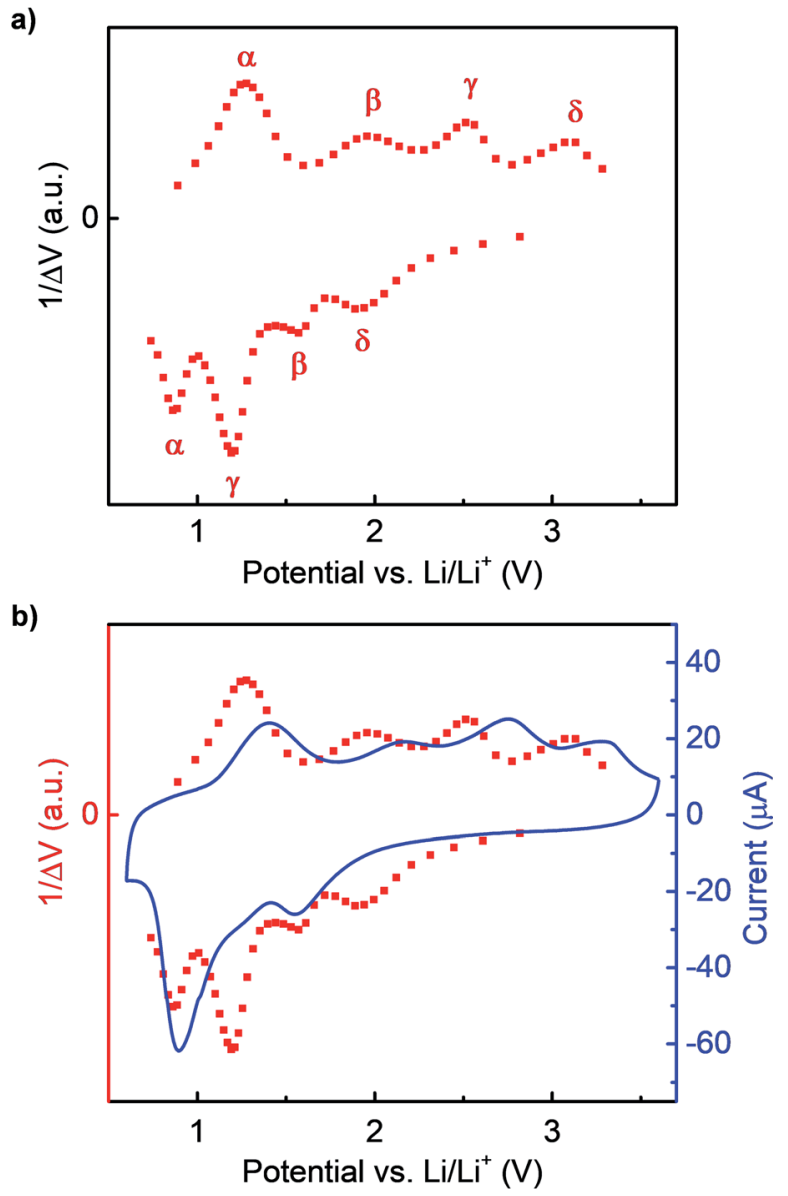

Fig. 2 (a) $1 / \Delta V$ vs. voltage curve extracted from GITT tests of a $\mathrm{RuO}_{2} /$ $\mathrm{LiPF}_{6} / \mathrm{Li}$ cell. The correspondence in the reactions leading to the delithiation and lithiation peaks, as defined by the labels $\alpha, \beta, \gamma$ and $\delta$, is explained in the text. (b) Comparison between the GITT curve and the $\mathrm{CV}$ curve obtained at $0.5 \mathrm{mV} \mathrm{s}^{-1}$.

CV plots. However, the GITT results clearly show four peaks during lithiation rather than three in the CV plot, confirming that the shoulder labeled $\beta$ in the $\mathrm{CV}$ plot results from a distinct reaction seen at about $1.6 \mathrm{~V}$ in the GITT plot and that the large peak at about $0.8 \mathrm{~V}$ in the $\mathrm{CV}$ plot is composed of two overlapping peaks that can be clearly distinguished in the GITT plot, at about $0.9 \mathrm{~V}$ and $1.3 \mathrm{~V}$.

Having confirmed that the same number of reactions occur during lithiation and delithiation, we now use CV curves to associate the lithiation peaks seen in the lithiation curve with the corresponding peaks in the delithiation curve. We performed CV scans with the same upper cutoff voltage $(3.6 \mathrm{~V})$ but with different lower cutoff voltages (Fig. 3a). We also performed CV scans with the same lower cutoff voltage $(0.6 \mathrm{~V})$ but with different upper cutoff voltages (Fig. 3b). The results are summarized in Fig. 3a and b, respectively.

As seen in Fig. 3a, when the lower cutoff voltage is $2.0 \mathrm{~V}$ (red) and $1.8 \mathrm{~V}$ (orange), before the first lithiation peak at around $1.6 \mathrm{~V}$, no obvious delithiation peak exists. When the lower cutoff voltage is further decreased to $1.4 \mathrm{~V}$, which is between the first lithiation peak and the shoulder at around $1.3 \mathrm{~V}$, a delithiation 
a)

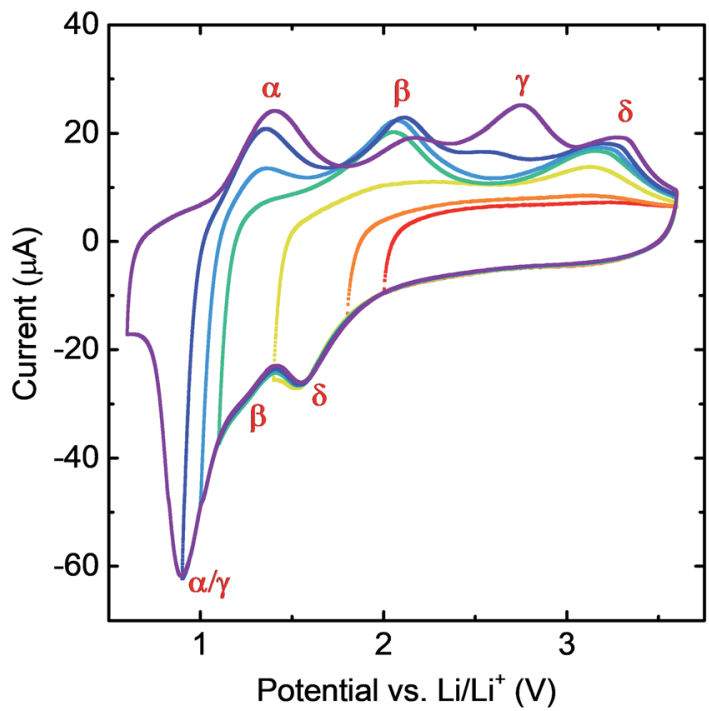

b)

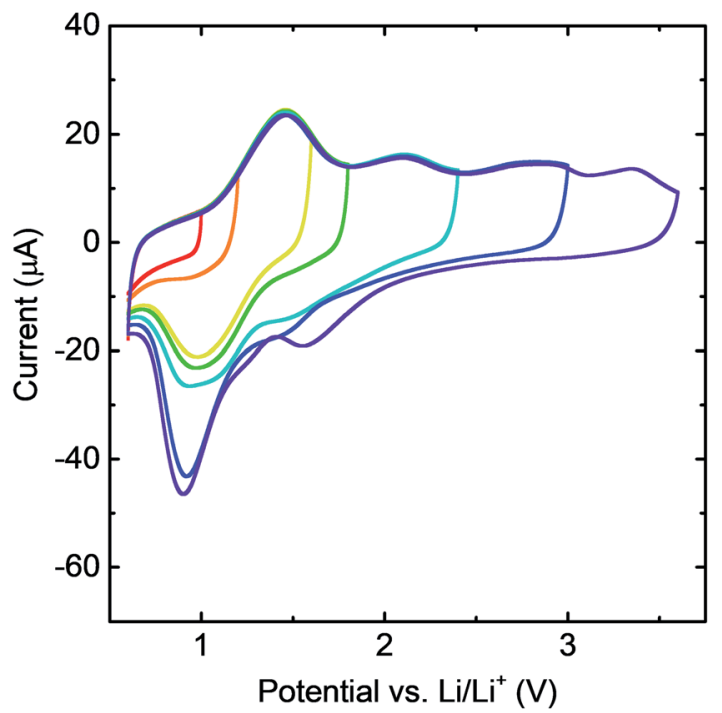

Fig. 3 Cyclic voltammograms of a $\mathrm{RuO}_{2} / \mathrm{LiPF}_{6} / \mathrm{Li}$ cell between (a) $3.6 \mathrm{~V}$ and different lower cutoff voltages and (b) $0.6 \mathrm{~V}$ and different upper cutoff voltages. All scan rates are $0.5 \mathrm{mV} \mathrm{s}^{-1}$. The peak labels shown in (a) are explained in the main text.

peak appears at around $3.2 \mathrm{~V}$. This shows that the lithiation peak at $1.6 \mathrm{~V}$ and the delithiation peak at $3.2 \mathrm{~V}$ correspond to the same reaction. When the lower cutoff voltage is further decreased to $1.1 \mathrm{~V}$ (green), after the shoulder $\beta$, a second delithiation peak appears at around $2.0 \mathrm{~V}$, which indicates that these peaks correspond to the same reaction. Finally, when the lower cutoff voltage was decreased to $1.0 \mathrm{~V}$ (azure), $0.9 \mathrm{~V}$ (blue) and $0.6 \mathrm{~V}$ (violet), we observe the appearance of two delithiation peaks at around $1.4 \mathrm{~V}$ and around $2.75 \mathrm{~V}$. That two delithiation peaks appear for this range of cutoff voltages is consistent with the fact that the lithiation peak at around $0.8 \mathrm{~V}$ in the CV curve was found to correspond to two peaks in the GITT curves.

These relationships are confirmed using CV scans with different upper cutoff voltages, shown in Fig. 3b. The lithiation peak at $0.8 \mathrm{~V}$ did not appear until the upper cutoff voltage was increased to $1.6 \mathrm{~V}$ (yellow), and thus this peak was identified as associated with reaction $\alpha$. The lithiation peak at $1.4 \mathrm{~V}$ appeared only after the upper cutoff voltage was increased to $2.4 \mathrm{~V}$ (cyan), and thus this peak was identified as associated with reaction $\beta$. Then, when the upper cutoff voltage was set at $3.0 \mathrm{~V}$ (blue), the intensity of the lithiation peak at $0.8 \mathrm{~V}$ increased significantly, indicating that this peak is also associated with reaction $\gamma$. Finally, for the fully delithiated sample (purple), the lithiation peak at around $1.6 \mathrm{~V}$ appeared, which implies that this peak is associated with reaction $\delta$.

To distinguish between the two overlapping low-voltage lithiation peaks, GITT scans with different lower cutoff voltages were performed, and the corresponding $1 / \Delta V \nu s$. voltage curves are shown in Fig. S9. $\dagger$ These results show that during lithiation, the reaction corresponding to $\gamma$ occurs at a higher voltage than that of reaction $\alpha$.

In summary, using GITT scans and CV scans with different lower cutoff and upper cutoff voltages, we confirmed that the lithiation $\mathrm{CV}$ peak at around $1.6 \mathrm{~V}$ corresponds to the delithiation peak at around $3.2 \mathrm{~V}$, and the lithiation shoulder at around $1.3 \mathrm{~V}$ corresponds to the delithiation peak at around $2.0 \mathrm{~V}$. The lithiation $\mathrm{CV}$ peak at around $0.8 \mathrm{~V}$ is a combination of two reaction peaks corresponding to the delithiation peaks at $1.4 \mathrm{~V}$ and $2.75 \mathrm{~V}$. When the kinetic effects that cause overlapping peaks associated with the $\alpha$ and $\gamma$ during lithiation were eliminated in GITT experiments, the lithiation reaction corresponding to the delithiation peak at $2.75 \mathrm{~V}$ is found to occur at a higher voltage than the reaction corresponding to the delithiation peak at $1.4 \mathrm{~V}$. These relationships between the delithiation and lithiation peaks have been indicated by the labels in Fig. 2a and 3a.

In addition, it can be concluded from GITT cycle curves (Fig. S1†) and the $1 / \Delta V v$ s. voltage curve (Fig. 2a) that reaction $\gamma$ contributed the most to the large voltage hysteresis of $\mathrm{RuO}_{2}$ during cycling. The hysteresis reached a magnitude of about $1.2 \mathrm{~V}$, even when a relaxation time of 1.5 hours was used between steps.

\subsection{Cross sectional transmission electron diffraction}

After determining the correspondence between lithiation and delithiation reactions shown in CV curves and deconvolving kinetic effects that lead to overlapping peaks using both GITT and CV experiments, ex situ characterizations using SAED, XPS and Raman spectroscopy on cycled thin film $\mathrm{RuO}_{2}$ samples were carried out. All samples were cycled at $0.5 \mathrm{mV} \mathrm{s}^{-1}$ until their cyclic voltammograms became stable. Different samples were then cycled at the same rate and stopped between different reaction peaks during the delithiation process, at the voltages (1), (2) and (3) shown in Fig. 4a.

Electron diffraction patterns (EDPs) were obtained from samples that were lithiated to the same lower cutoff voltage $0.6 \mathrm{~V}$ and then delithiated to the different voltages indicated in Fig. $4 \mathrm{a}, 1.8 \mathrm{~V}, 2.3 \mathrm{~V}, 3.0 \mathrm{~V}$, and $3.6 \mathrm{~V}$, corresponding to (1), (2), (3), and $\square$, respectively. The radial average intensity functions for each case can be found in Fig. S4. $\dagger$ Diffraction rings that can be indexed to Ru are observed in the fully lithiated sample ( $\mathbf{\square})$, as 
a)

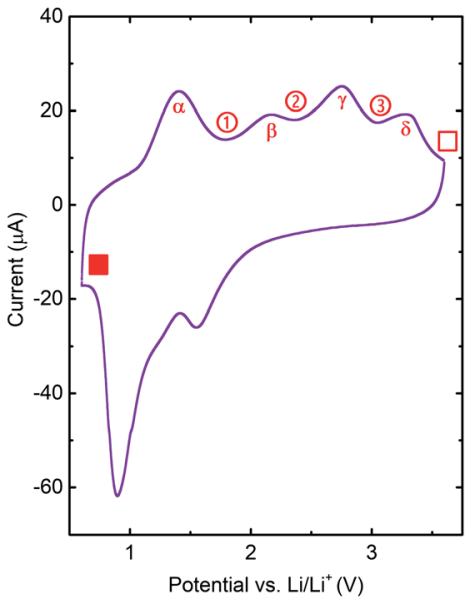

d)

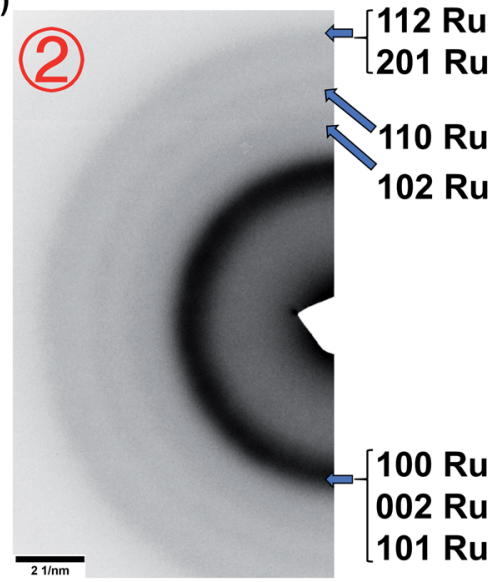

b)

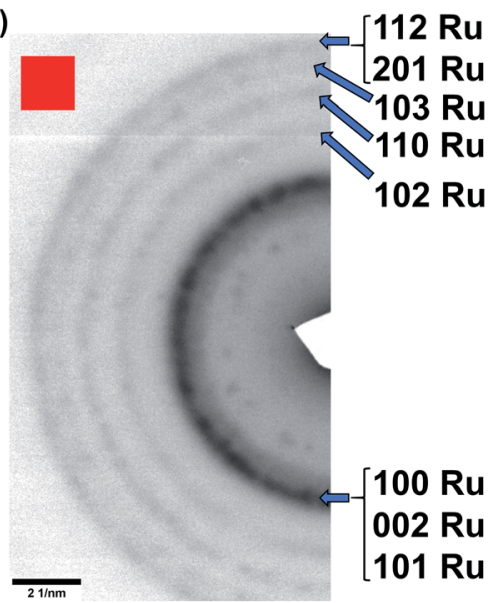

e)

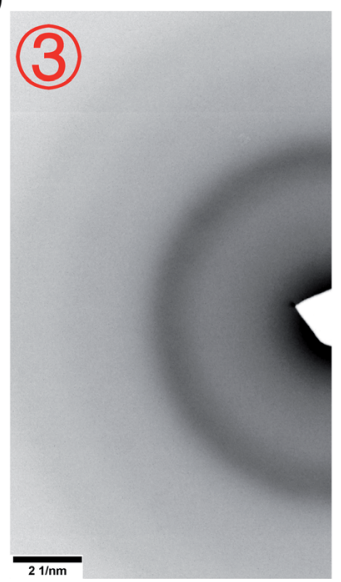

c)

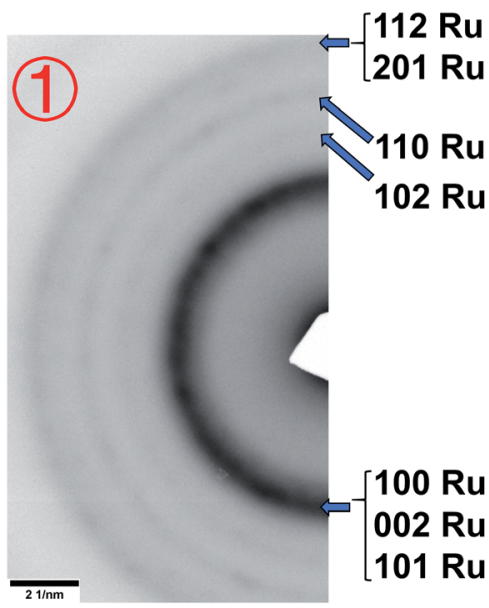

f)

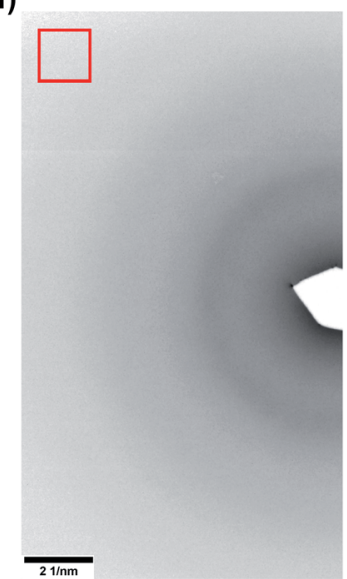

Fig. 4 (a) Labeled CV curve. (b) Electron diffraction pattern (EDP) for a sample that was lithiated to $0.6 \mathrm{~V}$ were first lithiated to $0.6 \mathrm{~V}$ and then delithiated to $1.8 \mathrm{~V}(1), 2.3 \mathrm{~V}(2), 3.0 \mathrm{~V}(3)$ or $3.6 \mathrm{~V}(\square)$.

shown in Fig. 4b, and the fully delithiated $\mathrm{RuO}_{2}(\square)$ is amorphous, as indicated by the broad halo seen in Fig. 4f. These results are consistent with previous in situ TEM studies. ${ }^{19}$ However, the (111) ring for $\mathrm{Li}_{2} \mathrm{O}$ was missing for the lithiated sample $(\boldsymbol{\square})$. This could be due to the high reactivity of $\mathrm{Li}_{2} \mathrm{O}$ as the fabrication of ex situ TEM samples inevitably included exposure to ambient, during which $\mathrm{Li}_{2} \mathrm{O}$ was likely to quickly degrade. For the sample delithiated to the intermediate state (1), after the first delithiation reaction $\alpha$, all $\mathrm{Ru}$ rings are still present (Fig. 4c and $\mathrm{S} 4 \dagger$ ), though with fewer detectable individual diffraction spots and with more diffuse rings, suggesting that the Ru grain size has decreased. ${ }^{32}$ After delithiation to state (2), a voltage higher than required for the second delithiation reaction $\beta, \mathrm{Ru}$ rings still exist (Fig. $4 \mathrm{~d}$ and $\mathrm{S} 4 \dagger$ ). Finally, after delithiation to a voltage above the delithiation peak $\gamma$, all $\mathrm{Ru}$ rings are missing except for a very weak remnant of the $\mathrm{Ru}(200)$ ring (Fig. 4e and $\mathrm{S} 4 \dagger$ ). These results show that metallic $\mathrm{Ru}$ forms in films lithiated to $0.6 \mathrm{~V}$ and remains present during delithiation to voltages higher than those of the $\alpha$ and $\beta$ peaks, and is substantially reduced only at voltages above those associated with the $\gamma$ reaction. Metallic Ru is presumably consumed in the conversion reaction to form $\mathrm{RuO}_{2}$. At a voltage above that associated with the reaction $\delta$, the fourth delithiation peak, the film is amorphous and is presumably fully converted to $\mathrm{RuO}_{2}$.

\subsection{X-ray photoelectron spectroscopy}

To support our findings from the EDPs, ex situ X-ray photoelectron spectra of $\mathrm{Ru} 3 \mathrm{~d}$ orbitals for all samples were acquired and are shown in Fig. 5, with curves fitted for a mixture of metallic Ru metal and $\mathrm{RuO}_{2} \cdot{ }^{33}$ At the beginning, when the film was fully lithiated at $0.6 \mathrm{~V}$ (Fig. 5a), the spectrum is dominated by metallic $\mathrm{Ru}$, with only a small fraction of the $\mathrm{Ru}$ involved in bonding with $\mathrm{O}$. This shows that during lithiation, $\mathrm{RuO}_{2}$ is converted to $\mathrm{Ru}$ metal, presumably through a reaction to form $\mathrm{Li}_{2} \mathrm{O}$. Metallic $\mathrm{Ru}$ continues to be the dominant form of $\mathrm{Ru}$ through the first and second delithiation peaks (Fig. 5b and c). It is only after the third delithiation reaction $\gamma$ (Fig. 5d), that $\mathrm{RuO}_{2}$ begins to dominate. This change suggests that the third delithiation peak $\gamma$ is associated with the reaction of metallic $\mathrm{Ru}$ with $\mathrm{Li}_{2} \mathrm{O}$ to form $\mathrm{RuO}_{2}$. These findings are consistent with the EDP results. In addition, the $\mathrm{RuO}_{2} / \mathrm{Ru}$ metal ratio after peak $\delta$ was slightly higher than that before peak $\delta$, which implies that there is a change of oxidation state during peak $\delta$. 
a)

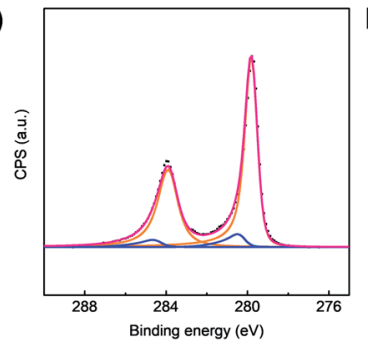

c)

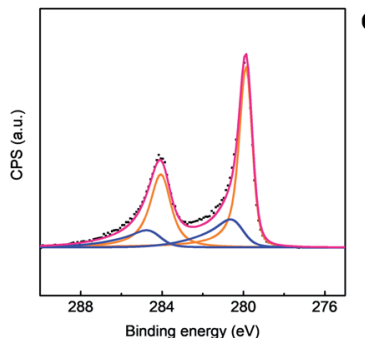

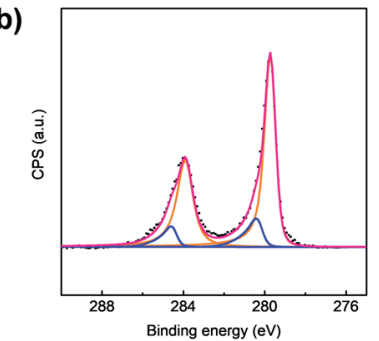

d)

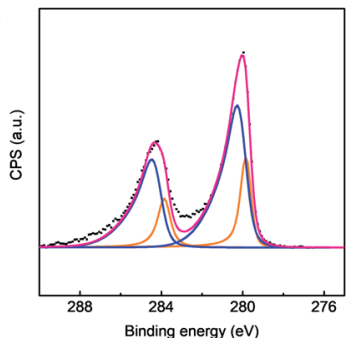

e)

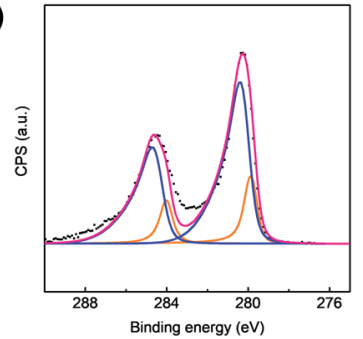

Fig. 5 (a) Ru 3d XPS spectrum for a sample lithiated to $0.6 \mathrm{~V}$ ( $\square$ ). (b-e) XPS spectra for samples first lithiated to $0.6 \mathrm{~V}$ and then delithiated to $1.8 \mathrm{~V}(1)$ ), $2.3 \mathrm{~V}($ (2)), 3.0 V (3) or $3.6 \mathrm{~V}$ ( $\square$ ) (black dots are experimental data, blue lines are $\mathrm{RuO}_{2}$ components, orange lines are $\mathrm{Ru}$ components, and pink lines are fitted curves).

\subsection{Raman spectroscopy}

Raman spectroscopy was also carried out to investigate the reactions that occur during delithiation, and by inference, lithiation. Data are shown in Fig. 6a, with the lowest curve corresponding to a sample in the fully lithiated state $(0.6 \mathrm{~V})$, and the ascending sequence of curves corresponding to samples that were delithiated to increasing voltages (as indicated in Fig. 4a). A peak is observed at about $240 \mathrm{~cm}^{-1}$ for all samples. The origin of this peak is unclear but it appears to minimally change at different stages of delithiation. This peak was therefore used as a reference peak for comparisons of the intensity of other peaks for samples delithiated to different voltages.

The peak at a Raman shift of around $191 \mathrm{~cm}^{-1}$ is associated with metallic $\mathrm{Ru} .{ }^{34}$ To confirm this, we obtained a separate Raman spectrum for a $10 \mathrm{~nm}$-thick single crystal Ru film, Fig. S5, $\uparrow$ for which a very strong peak at $191 \mathrm{~cm}^{-1}$ was seen. To focus on the intensity of this peak as a function of the degree of delithiation, the contribution from the peak at $240 \mathrm{~cm}^{-1}$ was minimized by subtracting the Raman spectrum of a fully delithiated sample $(\square)$, in which no metallic Ru exists, from the spectra of samples delithiated to different extents ( $\mathbf{\square}$, (1), (2) and (3). The results between $150 \mathrm{~cm}^{-1}$ and $250 \mathrm{~cm}^{-1}$ are shown in Fig. 6b. As the sputtered $\mathrm{RuO}_{2}$ films were delithiated, the peak at $191 \mathrm{~cm}^{-1}$ remained sharp after the first delithiation

a)

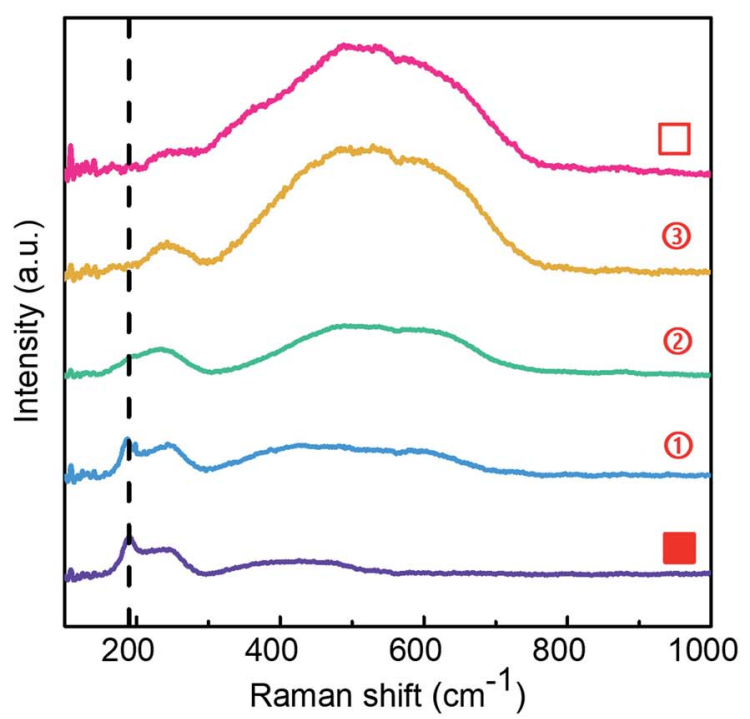

b)

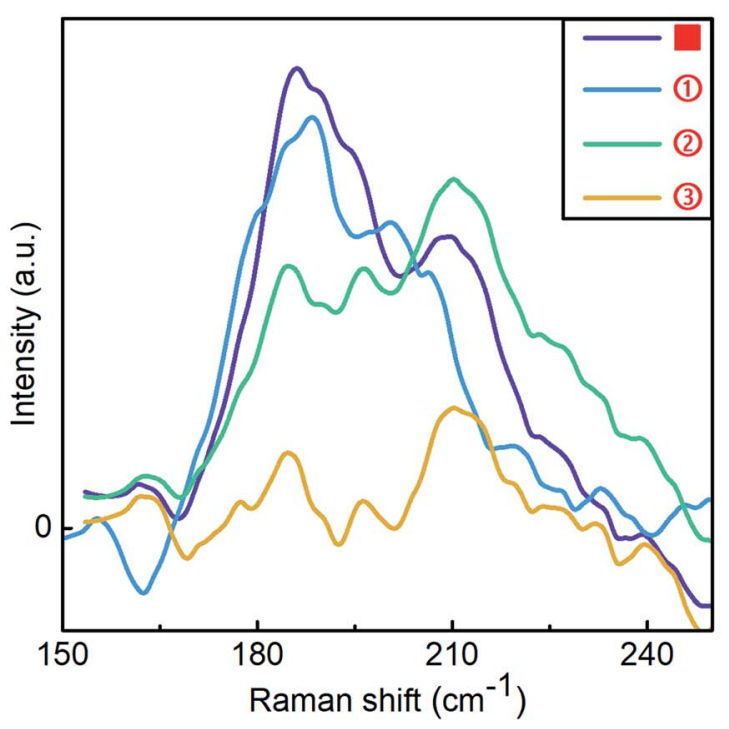

Fig. 6 (a) Raman spectra for samples lithiated to $0.6 \mathrm{~V}$, lower curve, and delithiated to different states (see Fig. 4a). (b) Raman spectra of samples at different lithiated states ( $\boldsymbol{\square}$, (1), (2) and (3)), between $150 \mathrm{~cm}^{-1}$ and $250 \mathrm{~cm}^{-1}$, after subtraction of the Raman spectrum of the fully delithiated sample $(\square)$.

peak $\alpha$ (1). This peak became a bit weaker but broader after the second delithiation peak $\beta$ (2), which indicates that the average grain size of the metallic Ru was smaller. ${ }^{35}$ After the third delithiation peak $\gamma$ (3), the Raman peak at about $191 \mathrm{~cm}^{-1}$ mostly disappears, showing that the amount of metallic $\mathrm{Ru}$ decreased significantly during the reaction $\gamma$.

As the degree of delithiation is increased, a collection of peaks develop between $400 \mathrm{~cm}^{-1}$ and $700 \mathrm{~cm}^{-1}$, which are thought to be associated with amorphous $\mathrm{RuO}_{2},{ }^{36}$ as the Raman spectrum of single crystal $\mathrm{RuO}_{2}$ consists of three peaks at 528, 646 and $716 \mathrm{~cm}^{-1}$. The relative intensity of these Raman peaks increased most significantly in association with CV peak $\gamma$ (going from (2) to (3), which suggests that the amount of $\mathrm{RuO}_{2}$ significantly increases during this reaction. These findings are 
consistent with the conclusions drawn based on EDP and XPS results.

\subsection{Surface morphology and chemistry}

In addition to the above characterizations, SEM and optical microscope images were captured and are shown in the ESI section as Fig. S6 and S7. $\uparrow$ The surface of the electrode was observed to be covered by a layer before the delithiation peak $\beta$ (for samples $\square$ and (1) but this layer was mostly removed after peak $\beta$ (for samples (2), (3) and $\square$ ). These results suggest that during delithiation, reaction $\beta$ involves the decomposition of a surface layer.

Ex situ energy dispersive X-ray spectroscopy (EDS) analysis also supports this hypothesis. Atomic percentages calculated using spectra collected from surfaces of the samples are listed in Table 1 (carbon not included). Before reaction $\beta$ (samples and (1)), a large amount of $\mathrm{F}$ was found on the surface. After reaction $\beta$ (samples (2), (3) and $\square$ ), the concentration of $\mathrm{F}$ decreased substantially. F cannot originate from the pristine $\mathrm{RuO}_{2}$ films, ambient air or moisture, and any residual salt on the sample surface was rinsed off using solvent before observations were made. However, $\mathrm{F}$ is commonly found within SEI films for electrolytes containing $\mathrm{LiPF}_{6} \cdot{ }^{\mathbf{1 5 , 4 1 , 4 2}}$ These results suggest that the decomposed surface layer was an SEI layer on $\mathrm{RuO}_{2}$.

\subsection{In situ EIS}

To further test this hypothesis, in situ EIS measurements were carried out and the results are summarized in Fig. 7. All the EIS measurements were carried out on freshly deposited thin-film $\mathrm{RuO}_{2}$ samples after ten CV cycles. From Fig. 7, it is seen that before the second delithiation peak $\beta$ (samples $\square$ and (1), an extra arc exists in the high-frequency range $(>50 \mathrm{~Hz}$, small $\operatorname{Re}(Z)$ ). This arc disappeared after peak $\beta$, (samples (2), (3) and $\square)$.

This also suggests that the second delithiation peak $\beta$ is related to the decomposition of the observed surface layer. As previous studies have revealed that a high-frequency $(>30 \mathrm{~Hz})$ arc is often associated with SEI layers on the surfaces of other electrode materials, ${ }^{37-40}$ this in situ result further supports the hypothesis that the delithiation peak $\beta$ can be attributed to the decomposition of an surface layer.

\subsection{PITT tests}

PITT tests were performed within the voltage range of the conversion reaction $\gamma(2-3 \mathrm{~V})$ to study its kinetic behavior. Part

Table 1 Atomic percentages of different elements on the electrode surfaces calculated from EDS spectra

\begin{tabular}{lllcl}
\hline Sample & $\mathrm{Ru}$ & $\mathrm{O}$ & $\mathrm{F}$ & $\mathrm{P}$ \\
\hline$\square$ & 10.8 & 34.8 & 41.1 & 2.1 \\
(1) & 17.8 & 15.1 & 59.8 & 1.9 \\
(2) & 30.8 & 47.2 & 15.6 & 0 \\
(3) & 27.2 & 72.8 & 0 & 0 \\
$\square$ & 23.3 & 66.00 & 4.8 & 0
\end{tabular}

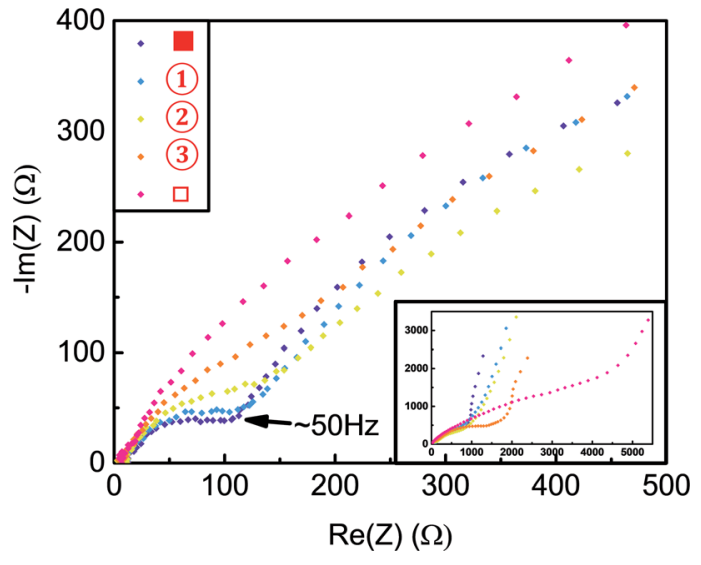

Fig. 7 Nyquist plots of the potentiostatic impedance of different delithiated samples. The inset shows the entire dataset.

of the PITT data is shown in Fig. 8 and the complete data from $2 \mathrm{~V}$ to $2.95 \mathrm{~V}$ can be found in Fig. S10. $\uparrow$ The black curves show the current as a function of time after each voltage step, and the blue lines show the applied voltages during each step. As can be seen in Fig. 8, the current during each step decreases monotonically without a local maximum. This not only applies to the data shown in the figure, but also to data collected for various step sizes, holding times and voltage ranges.

PITT data can be used to distinguish between diffusionlimited reactions and phase-transition-limited reactions. ${ }^{43-45}$ When the reaction is diffusion-limited, the current decays monotonically during each voltage step. In contrast, when the reaction is phase-transition-limited, the current first decays, then increases and reaches a maximum, and finally decays again.

The monotonic decay observed during PITT measurements of $\mathrm{RuO}_{2}$ in the voltage range associated with the CV peak for $\gamma$ suggests that the conversion reaction is diffusion-limited, not limited by the phase-transition. To characterize the length scale over which these diffusive processes occurred, CV scans of samples with different thicknesses were also performed

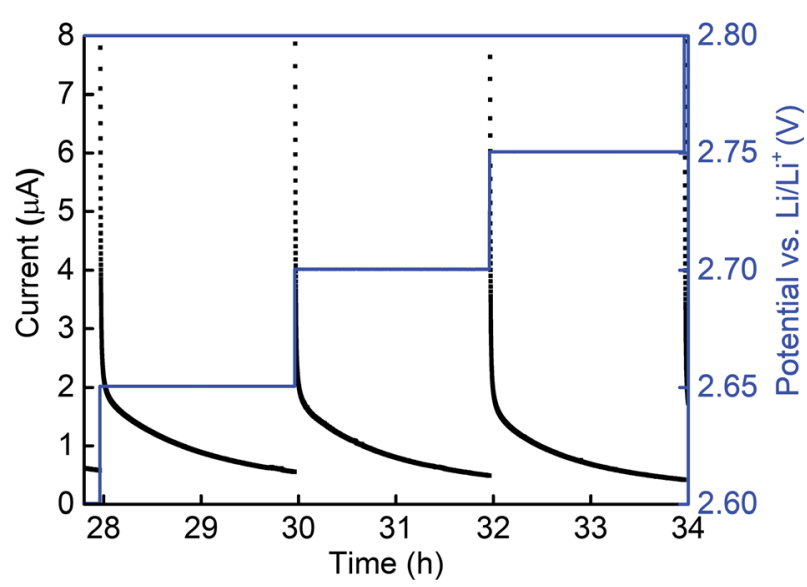

Fig. 8 Current and voltage as a function of time during PITT test of a $\mathrm{RuO}_{2} / \mathrm{LiPF}_{6} / \mathrm{Li}$ cell. The steps shown are $2.65 \mathrm{~V}, 2.70 \mathrm{~V}$ and $2.75 \mathrm{~V}$. 


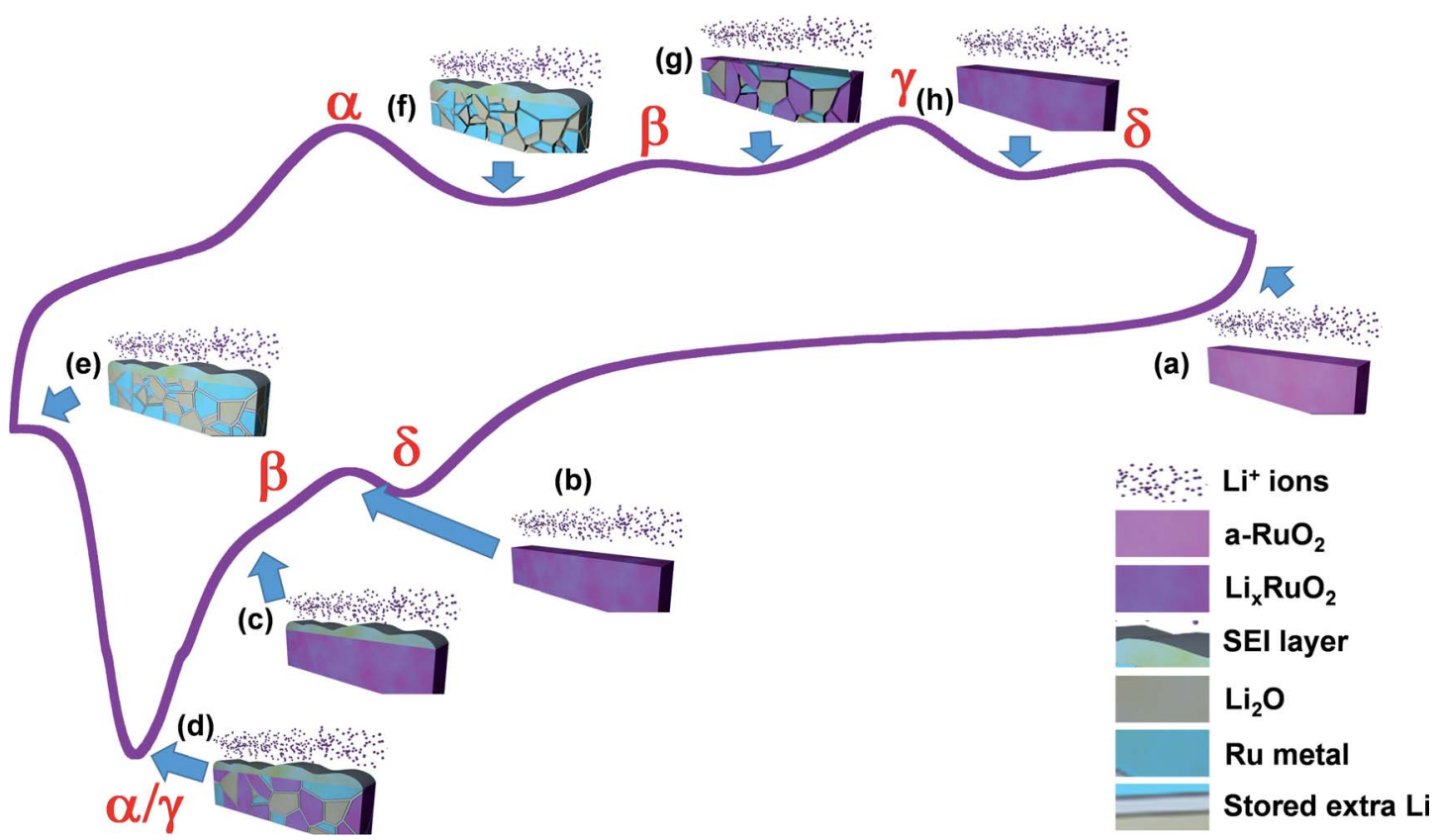

Fig. 9 Phase evolution and corresponding reactions during cyclic lithiation and delithiation of $\mathrm{RuO}_{2}$ films. (a) $\mathrm{Amorphized} \mathrm{RuO}_{2}$ after multiple initial cycles. (b) After the first lithiation reaction $\delta$, transition to a new $\mathrm{Li}_{x} \mathrm{RuO}_{2}$ phase has occurred. (c) During the second lithiation reaction $\beta$, an SEI layer forms on the surface. (d) During further lithiation, the conversion reaction $\gamma$ takes place and excess Li is stored between nanosized particles, $\alpha$. (e) Fully lithiated $\mathrm{Li} / \mathrm{Ru} / \mathrm{Li}_{2} \mathrm{O}$ film. (f) During the first delithiation reaction $\alpha$, inter-cluster Li has been removed. (g) After the second delithiation reaction $\beta$, the SEI layer on the surface has disappeared. (h) After the third delithiation reaction $\gamma$, the film has converted back to $\mathrm{Li}_{x} \mathrm{RuO}_{2}$. (a) After the fourth delithiation peak $\delta$, Li was removed from $\mathrm{Li}_{x} \mathrm{RuO}_{2}$ to form amorphous $\mathrm{RuO}_{2}$.

(Fig. S3†). The positions of all four lithiation and delithiation peaks did not shift significantly even when the thickness was doubled, suggesting that the rate-limiting diffusive processes operate at length-scales that are small compared to the film thickness, and that they occur uniformly through the film thickness.

\subsection{Discussion}

During delithiation, reaction $\alpha$ happens first. $\mathrm{Ru}$ metal was found both before and after this reaction. Previous studies of the reactions that occurred during the first lithiation of pristine crystalline $\mathrm{RuO}_{2}$ have indicated that during the late stages of lithiation, excess $\mathrm{Li}$ can be stored between nanosized $\mathrm{Ru}$ and $\mathrm{Li}_{2} \mathrm{O}$ phases. ${ }^{21,23,24}$ Thus, we propose that reaction $\alpha$ is:

Excess $\mathrm{Li}$ in $\mathrm{Ru} / \mathrm{Li}_{2} \mathrm{O}$ mixture $\rightarrow \mathrm{Li}$ (counter electrode) $+\mathrm{Ru} / \mathrm{Li}_{2} \mathrm{O}$ mixture.

This is consistent with the smaller overpotential shown in the $\mathrm{CV}$ curve, as the desorption of Li doesn't involve chemical reactions and is kinetically easier.

The nature of the second delithiation reaction $\beta$ is not as clear as that of the other reactions. As discussed above, ex situ microscopy shows clear evidence of a surface layer when films are lithiated to voltages below the $\beta$ peak, and that this layer is not present on films that have been delithiated beyond the $\beta$ peak. This surface layer might be the result of air exposure.
However, in situ EIS measurements also show evidence for reversible formation of a surface layer in the voltage ranges associated with the $\beta$ reaction. Also, EDS analysis of the layers shows a high level of $\mathrm{F}$. These results suggest that reaction $\beta$ might be associated with reversible formation of an SEI layer. If this is the case, reaction $\beta$ would be:

$$
\mathrm{SEI}(\mathrm{Li}) \rightarrow \mathrm{Li}(\text { counter electrode) + electrolyte }
$$

This is consistent with the GITT/CV results, in which the sequence of lithiation reactions $(\alpha \rightarrow \gamma \rightarrow \beta \rightarrow \delta)$ is different from the sequence of delithiation reactions $(\alpha \rightarrow \beta \rightarrow \gamma \rightarrow \delta)$, which implies that the delithiation reaction $\beta$ was independent of the other reactions.

As $\mathrm{Ru}$ metal was present before the third delithiation reaction $\gamma$ but not after, we propose that the reaction corresponding to this peak involves the oxidation of Ru metal:

$$
2 \mathrm{Li}_{2} \mathrm{O}+\mathrm{Ru} \rightarrow \mathrm{Li}_{x} \mathrm{RuO}_{2}+(4-x) \mathrm{Li} \text { (counter electrode). }
$$

This can also account for the fact that reaction $\alpha$ follows reaction $\gamma$ during lithiation, as the small clusters of $\mathrm{Ru}$ and $\mathrm{Li}_{2} \mathrm{O}$ only form when the conversion reaction $\gamma$ happens, during which excess Li can diffuse and reside between these phases.

Finally, given earlier studies of shallow lithiation of powderbased amorphous $\mathrm{RuO}_{2},{ }^{17,18}$ we suggest that the change in oxidation state indicated by the slight shift of $\mathrm{Ru} 3 \mathrm{~d}$ peaks in 
XPS spectra is associated with reaction peak $\delta$. That is, Li alloys with amorphous $\mathrm{RuO}_{2}$ and forms an amorphous $\mathrm{Li}_{x} \mathrm{RuO}_{2}$ phase, so that the reaction leading to the fourth delithiation peak $\delta$ would be:

$$
\mathrm{Li}_{x} \mathrm{RuO}_{2} \rightarrow \mathrm{RuO}_{2}+x \mathrm{Li} \text { (counter electrode). }
$$

Given the correspondence between lithiation and delithiation reactions that was determined from CV/GITT tests, the sequence of reactions during lithiation (from high to low voltages) would therefore be:

$$
\begin{gathered}
\mathrm{RuO}_{2}+x \mathrm{Li} \text { (counter electrode) } \rightarrow \mathrm{Li}_{x} \mathrm{RuO}_{2} \\
\mathrm{Li}(\text { counter electrode) }+ \text { electrolyte } \rightarrow \text { SEI (Li) } \\
\mathrm{Li}_{x} \mathrm{RuO}_{2}+(4-x) \mathrm{Li} \text { (counter electrode) } \rightarrow 2 \mathrm{Li}_{2} \mathrm{O}+\mathrm{Ru}
\end{gathered}
$$

and (3) is followed by,

$\mathrm{Li}$ (counter electrode) $+\mathrm{Ru} / \mathrm{Li}_{2} \mathrm{O}$ mixture $\rightarrow$ excess $\mathrm{Li}$ in $\mathrm{Ru} / \mathrm{Li}_{2} \mathrm{O}$ mixture

The proposed sequence for phase evolution during both lithiation and delithiation of $\mathrm{RuO}_{2}$ is summarized in Fig. 9.

By determining the reactions that occur during lithiation and delithiation of amorphous $\mathrm{RuO}_{2}$ thin films, it is concluded that the large voltage hysteresis of the conversion reaction contributes the most to the overall large voltage hysteresis, which significantly reduces the cycling energy efficiency. The conversion reaction during delithiation involves three kinetic sub-steps: nucleation of a new phase, $\mathrm{Li}_{x} \mathrm{RuO}_{2}$, at the interfaces between $\mathrm{Ru}$ and $\mathrm{Li}_{2} \mathrm{O}$ clusters, local diffusion of $\mathrm{O}$ or $\mathrm{Ru}$ during growth of the new phase, and long-range diffusion of Li through the thin film. PITT results suggest that the nucleation and growth of the new phase is not the rate-limiting step. Moreover, the CV curves of $\mathrm{RuO}_{2}$ samples with different thicknesses (Fig. S3†) show no significant shift of reaction peak $\gamma$, although the thickness was more than doubled (from $160 \mathrm{~nm}$ to $400 \mathrm{~nm}$ ). This implies that long-range diffusion of Li through the film is not the cause of the large voltage hysteresis. Consequently, the large voltage hysteresis of the conversion reaction is most likely due to local diffusion of $\mathrm{O}$ or $\mathrm{Ru}$ atoms.

\section{Conclusions}

Using a range of electrochemical and structural characterizations of multiply-cycled $\mathrm{RuO}_{2}$ films, we show that four reactions occur during lithiation: formation of amorphous $\mathrm{Li}_{x} \mathrm{RuO}_{2}$ by insertion of $\mathrm{Li}$ into amorphous $\mathrm{RuO}_{2}$, formation of an SEI layer, a conversion reaction to form $\mathrm{Li}_{2} \mathrm{O}$ and $\mathrm{Ru}$ metal from $\mathrm{Li}_{x} \mathrm{RuO}_{2}$, and insertion of metallic $\mathrm{Li}$ between nanosized $\mathrm{Ru}$ and $\mathrm{Li}_{2} \mathrm{O}$ particles. The same four reactions occur during delithiation, but not simply in reverse order. First $\mathrm{Li}$ is removed from between $\mathrm{Ru}$ and $\mathrm{Li}_{2} \mathrm{O}$ particles, the SEI layer that was formed during lithiation then reversibly dissociates, $\mathrm{Ru}$ and $\mathrm{Li}_{2} \mathrm{O}$ are then converted to $\mathrm{Li}_{x} \mathrm{RuO}_{2}$ and $\mathrm{Li}$ is removed from $\mathrm{Li}_{x} \mathrm{RuO}_{2}$ to form $\mathrm{RuO}_{2}$.

The largest portion of the $\mathrm{Li}$ capacity of $\mathrm{RuO}_{2}$ electrodes is associated with the conversion reaction, and the potentials at which $\mathrm{Li}_{2} \mathrm{O}$ and $\mathrm{Ru}$ form from $\mathrm{Li}_{x} \mathrm{RuO}_{2}$ during lithiation and at which the reverse reaction occurs during delithiation are separated by about $1.2 \mathrm{~V}$ in galvanostatic intermittent titrations. This leads to a hysteretic effect that causes poor cycling efficiency for $\mathrm{RuO}_{2}$, and by analogy, likely for other conversion electrode materials. We suggest that the high overpotentials required for these reactions are due to kinetic limitations associated with short range diffusion.

The methodologies used here for analysis of reactions that lead to high capacities in thin film electrodes, especially conversion-reaction electrodes, provide useful complements to analyses of powder-based materials. These studies also provide an important background for ongoing efforts to improve performance of $\mathrm{RuO}_{2}$ electrodes for thin film batteries.

\section{Conflicts of interest}

There are no conflicts to declare.

\section{Acknowledgements}

This research was supported by the Skoltech Center for Electrochemical Energy Storage as part of the Skoltech-MIT program. It was also supported by the National Research Foundation, Prime Minister's Office, Singapore, under its Campus for Research Excellence and Technological Enterprise (CREATE) program, through the Singapore-MIT Alliance for Research and Technology (SMART), Low Energy Electronic Systems (LEES) Interdisciplinary Research Group (IRG). We thank S. Ezzat and K. S. Coffey of the University of Central Florida for single-crystal $\mathrm{Ru}$ films. We are also grateful for helpful discussions with J. Miao, B. Wang, M. J. Chon and A. Weathers. This work made use of the MRL MRSEC Shared Experimental Facilities at MIT, supported by the National Science Foundation under award number DMR-1419807.

\section{Notes and references}

1 S. Ornes, Proc. Natl. Acad. Sci. U. S. A., 2016, 113, 1105911060.

2 V. Aravindan, Y.-S. Lee and S. Madhavi, Adv. Energy Mater., 2015, 5, 1402225.

3 M. H. Oh, T. Yu, S.-H. Yu, B. Lim, K.-T. Ko, M.-G. Willinger, D.-H. Seo, B. H. Kim, M. G. Cho, J.-H. Park, K. Kang, Y.-E. Sung, N. Pinna and T. Hyeon, Science, 2013, 340, 964968.

4 J. Jiang, Y. Li, J. Liu, X. Huang, C. Yuan and X. W. D. Lou, Adv. Mater., 2012, 24, 5166-5180.

5 S. M. Yuan, J. X. Li, L. T. Yang, L. W. Su, L. Liu and Z. Zhou, ACS Appl. Mater. Interfaces, 2011, 3, 705-709.

6 S. H. Yu, S. H. Lee, D. J. Lee, Y. E. Sung and T. Hyeon, Small, 2016, 12, 2146-2172. 
7 A. S. Hassan, K. Moyer, B. R. Ramachandran and C. D. Wick, J. Phys. Chem. C, 2016, 120, 2036-2046.

8 M. A. Lowe, J. Gao and H. D. Abruña, J. Mater. Chem. A, 2013, 1, 2094-2103.

9 P. G. Bruce, B. Scrosati and J. M. Tarascon, Angew. Chem., Int. Ed., 2008, 47, 2930-2946.

10 F. Wu and G. Yushin, Energy Environ. Sci., 2017, 10, 435-459.

11 M. Keppeler and M. Srinivasan, ChemElectroChem, 2017, 4, 2727-2754.

12 D. Perego, J. S. T. Heng, X. Wang, Y. Shao-Horn and C. V. Thompson, Electrochim. Acta, 2018, 283, 228-233.

13 J. Zhu, K. B. Yeap, K. Zeng and L. Lu, Thin Solid Films, 2011, 519, 1914-1922.

14 J. Zhu, K. Zeng and L. Lu, Metall. Mater. Trans. A, 2013, 44, 26-34.

15 P. Balaya, H. Li, L. Kienle and J. Maier, Adv. Funct. Mater., 2003, 13, 621-625.

16 T. Ohzuku, J. Electrochem. Soc., 1990, 137, 3004.

17 D. Muñoz-Rojas, M. Casas-Cabanas and E. Baudrin, Solid State Ionics, 2010, 181, 536-544.

18 O. Delmer, P. Balaya, L. Kienle and J. Maier, Adv. Mater., 2008, 20, 501-505.

19 K. E. Gregorczyk, Y. Liu, J. P. Sullivan and G. W. Rubloff, ACS Nano, 2013, 7, 6354-6360.

20 M. Mao, A. Nie, J. Liu, H. Wang, S. X. Mao, Q. Wang, K. Li and X.-X. Zhang, Nanotechnology, 2015, 26, 125404.

21 A. S. Hassan, A. Navulla, L. Meda, B. R. Ramachandran and C. D. Wick, J. Phys. Chem. C, 2015, 119, 9705-9713.

22 Y. Y. Hu, Z. Liu, K. W. Nam, O. J. Borkiewicz, J. Cheng, X. Hua, M. T. Dunstan, X. Yu, K. M. Wiaderek, L. S. Du, K. W. Chapman, P. J. Chupas, X. Q. Yang and C. P. Grey, Nat. Mater., 2013, 12, 1130-1136.

23 Y. Kim, S. Muhammad, H. Kim, Y. H. Cho, H. Kim, J. M. Kim and W. S. Yoon, ChemSusChem, 2015, 8, 2378-2384.

24 E. Bekaert, P. Balaya, S. Murugavel, J. Maier and M. Ménétrier, Chem. Mater., 2009, 21, 856-861.

25 J. Cabana, L. Monconduit, D. Larcher and M. R. Palacín, Adv. Mater., 2010, 22, 170-192.

26 Y. Oumellal, A. Rougier, G. A. Nazri, J. M. Tarascon and L. Aymard, Nat. Mater., 2008, 7, 916-921.
27 F. Wang, R. Robert, N. A. Chernova, N. Pereira, F. Omenya, F. Badway, X. Hua, M. Ruotolo, R. Zhang, L. Wu, V. Volkov, D. Su, B. Key, M. Stanley Whittingham, C. P. Grey, G. G. Amatucci, Y. Zhu and J. Graetz, J. Am. Chem. Soc., 2011, 133, 18828-18836.

28 L. Li, R. Jacobs, P. Gao, L. Gan, F. Wang, D. Morgan and S. Jin, J. Am. Chem. Soc., 2016, 138, 2838-2848.

29 I. Ullah, Y. Xu, W. ur Rehman, Y. Zhang, C. Wang and L. Li, J. Alloys Compd., 2019, 802, 591-599.

30 W. Dreyer, J. Jamnik, C. Guhlke, R. Huth, J. Moškon and M. Gaberšček, Nat. Mater., 2010, 9, 448-453.

31 V. A. Sethuraman, V. Srinivasan and J. Newman, J. Electrochem. Soc., 2013, 160, A394-A403.

32 A. L. Patterson, Phys. Rev., 1939, 56, 978-982.

33 D. J. Morgan, Surf. Interface Anal., 2015, 47, 1072-1079.

34 M. Lewandowski, B. Scheibe, T. Vasileiadis, N. Michalak, Z. Miłosz, R. Ranecki, S. Mielcarek, T. Luciński and S. Jurga, Surf. Coat. Technol., 2015, 271, 87-91.

35 I. H. Campbell and P. M. Fauchet, Solid State Commun., 1986, 58, 739-741.

36 S. Y. Mar, C. S. Chen, Y. S. Huang and K. K. Tiong, Appl. Surf. Sci., 1995, 90, 497-504.

37 S. S. Zhang, K. Xu and T. R. Jow, Electrochim. Acta, 2006, 51, 1636-1640.

38 L. Wang, J. Zhao, X. He, J. Gao, J. Li, C. Wan and C. Jiang, Int. J. Electrochem. Sci., 2012, 7, 345-353.

39 M. D. Levi, J. Electrochem. Soc., 1999, 146, 1279.

40 R. Yazami and D. Guérard, J. Power Sources, 1993, 43, 39-46. 41 A. Wang, S. Kadam, H. Li, S. Shi and Y. Qi, npj Comput. Mater., 2018, 4, 15.

42 N. Takenaka, Y. Suzuki, H. Sakai and M. Nagaoka, J. Phys. Chem. C, 2014, 118, 10874-10882.

43 J. Miao, B. Wang and C. V. Thompson, Phys. Rev. Mater., 2020, 4, 1-10.

44 N. Meethong, Y. H. Kao, W. C. Carter and Y. M. Chiang, Chem. Mater., 2010, 22, 1088-1097.

45 F. Y. Fan, W. C. Carter and Y. M. Chiang, Adv. Mater., 2015, 27, 5203-5209. 